\title{
Using Fluid Bed Granulation to Improve the Dissolution of Poorly Water-Soluble Drugs
}

\author{
Andrea Ikeda Takahashi, Felipe Rebello Lourenço, Marcelo Dutra Duque, Vladi Olga \\ Consiglieri and Humberto Gomes Ferraz \\ Departamento de Farmácia; Faculdade de Ciências Farmacêuticas; Universidade de São Paulo; São Paulo - SP - \\ Brasil
}

\begin{abstract}
In this study, fluid bed granulation was applied to improve the dissolution of nimodipine and spironolactone, two very poorly water-soluble drugs. Granules were obtained with different amounts of sodium dodecyl sulfate and croscarmellose sodium and then compressed into tablets. The dissolution behavior of the tablets was studied by comparing their dissolution profiles and dissolution efficiency with those obtained from physical mixtures of the drug and excipients subjected to similar conditions. Statistical analysis of the results demonstrated that the fluid bed granulation process improves the dissolution efficiency of both nimodipine and spironolactone tablets. The addition of either the surfactant or the disintegrant employed in the study proved to have a lower impact on this improvement in dissolution than the fluid bed granulation process.
\end{abstract}

Key words: fluid bed, granulation, dissolution efficiency, nimodipine, spironolactone

\section{INTRODUCTION}

Over the last decade, there has been an increase in drugs with high lipophilicity and poor watersolubility. These physicochemical characteristics lead to problematic biopharmaceutical properties, such as slow or incomplete absorption, which may impair bioavailability. A drug must dissolve in physiological intestinal fluids and be adequately absorbed in order to result in successful clinical treatment (Murali Mohan Babu et al. 2002; Stegeman et al. 2007). Several attempts have been made to enhance dissolution profiles and, therefore, the absorption and bioavailability of water-insoluble drugs. The use of water-soluble salts and polymorphic forms, as well as the formation of water-soluble molecular complexes, drug micronization, solid dispersions, coprecipitation, microencapsulation, spray-drying and co-grinding of drugs with commonly used excipients are some of the formulation tools that have been observed to enhance the dissolution characteristics of water-insoluble drugs (Leuner and Dressman 2000; Vogt et al. 2008a; Vogt et al. 2008b; Fahmy and Kassem 2008).

The granulation process is a pharmaceutical operation that obtains large granules from fine powders with the aim of improving flowability, appearance, mixing properties and the physical and chemical properties of these powders. In tablet manufacturing, a continuous production line, including several operations like granulation, drying and coating, can be executed in fluid bed equipment (Tardos et al. 1997; Iveson et al. 2001; Vervaet and Remon 2005).

The fluid bed granulation process consists of spraying a binder solution, dispersion or suspension onto a physical mixture, where

*Author for correspondence: sferraz@usp.br 
particles are suspended by air flow. These particles are wet by the binder solution, and liquid bridges are formed when they collide, leading to the acquisition of granules. Certain conditions, such as the spray rate and concentration of the binder solution, primary particle size, manufacturing processes, fluidizing air velocity and formulation can affect the granule microstructure and bed moisture level, leading to a lower rate of granule breakage (Bouffard et al. 2005; Ansari and Stepanek 2008).

However, despite the recognized utility of the fluid bed for improving the dissolution of poorly watersoluble drugs, there are no studies in which the drugs nimodipine and spironolactone have been processed using this technology for the production of granules and subsequent compression. It is important to emphasize that nimodipine and spironolactone are classified as class II and class II or IV, respectively, according to the Biopharmaceutics Classification System (Lindenberg et al. 2004; Papageorgiou et al. 2006). The purpose of this study was to investigate the feasibility of using the fluid bed technique to produce granules of these poorly water-soluble drugs, with improved dissolution rates. Dissolution efficiencies of the tablets produced from the fluid bed granules were compared to those obtained from the physical mixtures of the ingredients. Moreover, the role of sodium dodecyl sulfate and croscarmellose sodium in enhancing drug dissolution was evaluated.

\section{MATERIAL AND METHODS}

\section{Material}

Nimodipine and spironolactone were obtained from Labogen S/A Química Fina e Biotecnologia (Indaiatuba, Brazil) and Gerbrás Química Farmacêutica (Diadema, Brazil), respectively. Copovidone (Kollidon ${ }^{\circledR}$ VA64) was kindly donated by BASF S.A. (São Paulo, Brazil), and the other excipients, lactose M 200, talc, colloidal silicon dioxide, microcrystalline cellulose type 101, magnesium stearate, sodium dodecyl sulfate and croscarmellose sodium were all of pharmaceutical grade. The reagents sodium acetate, hydrochloric acid and glacial acetic acid were all of analytical grade.

\section{Preparation of nimodipine and spironolactone tablets}

Nimodipine and spironolactone granules containing $10.0 \%$ of the drug, $1.0 \%$ Kollidon $^{\circledR}$ VA64, $1.0 \%$ talc, $1.0 \%$ colloidal silicon dioxide and different amounts of sodium dodecyl sulphate ( 0 to $2.0 \%$ ) and croscarmellose sodium (0 to $1.0 \%$ ) were prepared. The formulations were completed to $100 \%$ with a 1:1 lactose-microcrystalline cellulose mixture. Granule compositions are shown in the Table 1. The binder dispersion was prepared by mixing the drug, Kollidon ${ }^{\circledR}$ VA64 and sodium dodecyl sulfate. The mixture was completed to $50.0 \mathrm{~mL}$ with distilled water, and this was placed in an Ultra Turrax $\mathrm{T} 25$ Digital Homogenizer (Ika Labortechnik, Staufen, Germany) for one minute, in order to disperse the material.

Initially, lactose, microcrystalline cellulose, talc and colloidal silicon dioxide were homogenized in a Mycrolab $^{\circledR}$ fluid bed system (Hüttlin, Steinen, Germany), for five minutes, under the following conditions: air volume: of $7.0 \mathrm{~m}^{3} \mathrm{~h}^{-1}$; inlet temperature: $50.0^{\circ} \mathrm{C}$; bottom spray pressure: 0.8 bar; microclimate: 0.4 bar; and filter clean pressure: 2.4 bar. The binder dispersion was added to the homogenized powders by means of a peristaltic pump (Watson-Marlow Bredel Pumps, England), at a rate of $10 \mathrm{rpm}$. The pump was connected to a $0.8 \mathrm{~mm}$ bottom spray nozzle for nimodipine, and a $0.6 \mathrm{~mm}$ nozzle for spironolactone. After applying the binder solution, the granules were dried in the fluid bed under the same conditions for five minutes. When applicable, croscarmellose sodium was added and mixed to the dried formulations. Finally, magnesium stearate was manually added to all formulations and they were stirred for two minutes. The physical mixtures were accurately prepared by weighing and mixing the same ingredients used in the preparation of granules. The final drug content in the granules was determined by means of a previously-validated UV spectrophotometric method, in order to define the tablet weight. 
Table 1 - Composition of the granules, physical mixtures and the tablets obtained from these materials.

\begin{tabular}{cccc}
\hline $\begin{array}{c}\text { Formulation (Granules } \\
\text { or Physical mixtures) }\end{array}$ & $\begin{array}{c}\text { Sodium dodecyl } \\
\text { sulfate }(\boldsymbol{\%})\end{array}$ & $\begin{array}{c}\text { Croscarmellose sodium* } \\
(\boldsymbol{\%})\end{array}$ & $\begin{array}{c}\text { Lactose-microcrystalline } \\
\text { cellulose mixture }(\mathbf{1 : 1})(\boldsymbol{\%})\end{array}$ \\
\hline 1 & 0 & 0 & 89.0 \\
2 & 0.5 & 0 & 88.5 \\
3 & 1.0 & 0 & 88.0 \\
4 & 2.0 & 0 & 87.0 \\
5 & 0 & 1.0 & 88.0 \\
6 & 0.5 & 1.0 & 87.5 \\
7 & 1.0 & 1.0 & 87.0 \\
8 & 2.0 & 1.0 & 86.0 \\
\hline
\end{tabular}

*Manually added after granulation process.

After being accurately weighed, the granules and the physical mixtures were compressed into tablets using a hydraulic press (Fred S. Carver inc., New Jersey, USA). A compression force of 0.5 ton was applied for one minute.

\section{Dissolution tests}

Dissolution tests were performed in a D-800 Logan Dissolution Tester (Logan Instruments Corp., New Jersey, USA) multi-bath dissolution test system. An assay of each tablet obtained from physical mixture (PM) and granules (G) was executed in triplicate $(\mathrm{n}=3)$. The nimodipine test was carried out according to the British Pharmacopoeia method (British Pharmacopoeia 2010), using apparatus 2 at $75 \mathrm{rpm}$ with $900 \mathrm{~mL}$ of acetate buffer, $\mathrm{pH} 4.5$, and $0.3 \%$ of sodium dodecyl sulfate. In order to prevent tablet floating, the dissolution test for spironolactone was conducted with apparatus 1 at $100 \mathrm{rpm}$ and a different pharmacopoeia method (United States Pharmacopeia 2009), in $0.1 \mathrm{M}$ hydrochloric acid and $0.1 \%$ of sodium dodecyl sulfate.

The medium, which was previously deaerated in an ultrasonic bath at $50^{\circ} \mathrm{C}$ and filtered through a $0.45 \mu \mathrm{m}$ membrane, was kept at $37 \pm 0.5^{\circ} \mathrm{C}$. The glass dissolution vessels were covered to minimize evaporation. Manual sampling aliquots of $10.0 \mathrm{~mL}$ were extracted at $2,5,10,15,20,30,45$ and 60 minutes for nimodipine, and at $3,5,10,15,20,30$, 45, 60 and 90 minutes for spironolactone.

A Beckman Coulter DU ${ }^{\circledR} 640$ UV-VIS Spectrophotometer (California, USA), with $1.0 \mathrm{~cm}$ quartz cells, adjusted to $360 \mathrm{~nm}$ for nimodipine and $238 \mathrm{~nm}$ for spironolactone, was used to record the samples. Other experiments, such as linearity, precision, recovery and absence of interference, were also conducted in order to achieve analytical validation (data not shown).
The dissolution profiles were compared through a model-independent method: dissolution efficiency (DE). The DE was calculated from the area under the dissolution curve versus time, calculated by means of the trapezoidal rule and expressed as a percentage of the rectangular area described by $100 \%$ dissolution in the same time. The results of DE were compared by means of ANOVA (analysis of variance) and were evaluated by interaction plot, using Minitab ${ }^{\circledR} 15$ statistical software to analyze the relationship between the DE values of the physical mixture and granules, and the concentration of sodium dodecyl sulfate, as well as the presence or absence of croscarmellose sodium.

\section{RESULTS AND DISCUSSION}

The dissolution profiles obtained from the tablets obtained from granules $(\mathrm{G})$ and from physical mixtures $(\mathrm{PM})$ are presented for nimodipine in Figure 1, and for spironolactone in Figure 2.

According to Figure 1A, nimodipine tablets obtained from physical mixtures (PM1, PM2, PM3 and PM4) did not present adequate dissolution profiles for immediate-release tablets. Drug release from formulations PM1, PM2 and PM3 was less than $40.0 \%$ within 60 minutes, while formulation PM4 released more than $80.0 \%$ of the drug over the same timeframe, probably as a result of the addition of $2.0 \%$ sodium dodecyl sulfate, which increased powder wettability (Vogt et al. 2008b).

The addition of a disintegrant to some formulations (PM5, PM6, PM7 and PM8), Figure $1 \mathrm{~B}$, led to an increase in the percentage of nimodipine dissolved, more than $85.0 \%$ within 60 minutes. But the dissolution profiles show that 
drug release from tablets obtained from physical mixtures containing the disintegrant are no faster than from tablets obtained from granules obtained by spraying the dispersed drug in the fluid bed equipment (Fig. 1B and Fig. 1D).
A

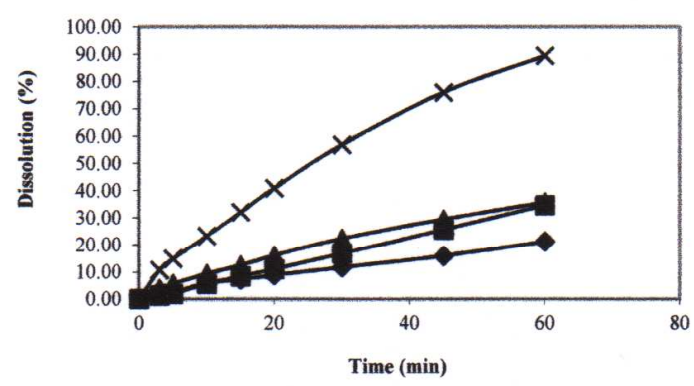

$\longrightarrow \mathrm{PM1} \rightarrow-\mathrm{PM} 2 \longrightarrow \mathrm{PM} 3 \longrightarrow \mathrm{PM} 4$

$\mathrm{C}$

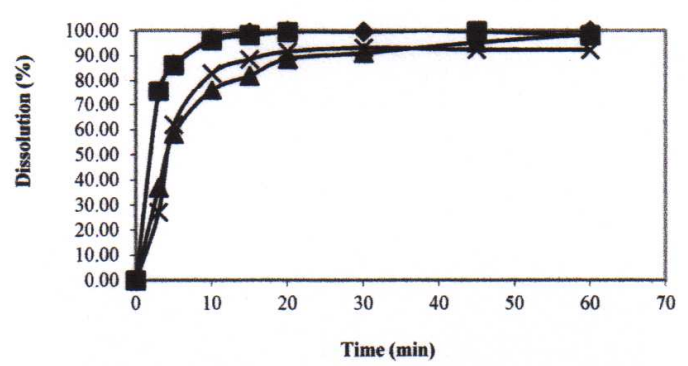

$\longrightarrow \mathrm{G}_{1} \longrightarrow \mathrm{G} 2_{2} \longrightarrow \mathrm{G}_{3} \longrightarrow \mathrm{G}_{4}$

\section{Nimodipine}

B
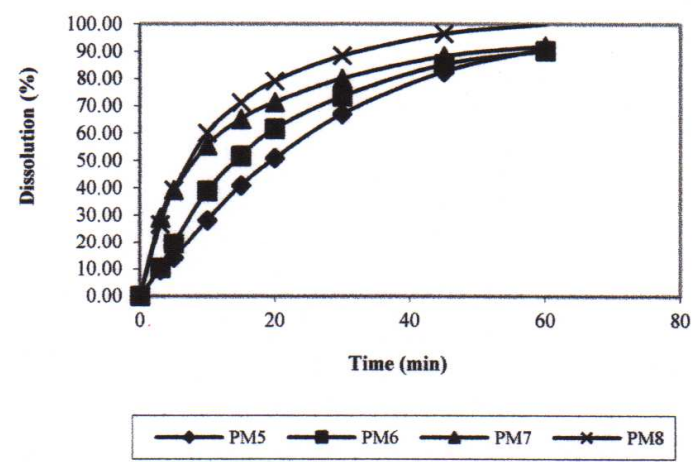

D

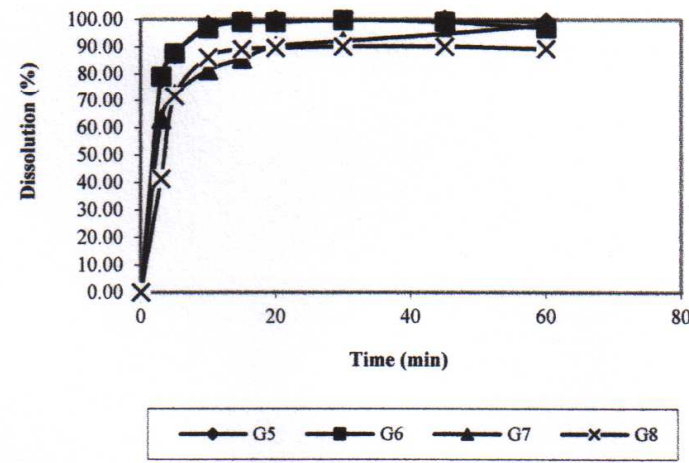

Figure 1 - Nimodipine dissolution profiles in acetate buffer solution, $\mathrm{pH} 4.5$, with $0.3 \%$ sodium dodecyl sulfate, using apparatus 2 at $75 \mathrm{rpm}$ for 60 minutes (PM= tablets obtained from physical mixtures, $\mathrm{G}=$ tablets obtained from granules).

Spironolactone tablet formulations obtained from physical mixtures (PM1, PM2, PM5 and PM6), both with and without the excipient croscarmellose sodium, presented similar behavior. On the other hand, the dissolution profiles of spironolactone formulations PM3 and PM4 versus PM7 and PM8, were different, as they exhibited a faster rate of drug release (as observed for PM7 and PM8 over the first 30 minutes) than can be explained by the use of $1.0 \%$ croscarmellose sodium alone. For all of these formulations, the release of spironolactone improved according to the amount of surfactant added, but drug release for the tablets obtained from physical mixtures was never greater than $65.0 \%$ within 90 minutes.

For spironolactone, the fluid bed process created granules which were made into tablets with significantly better dissolution profiles (more than $80.0 \%$ of the drug was dissolved) than the dissolution performance of the tablets obtained from physical mixtures, notwithstanding the presence of sodium dodecyl sulfate.

Upon analyzing the formulations containing fluid bed material processed (G1 versus G5, G2 versus G6, G3 versus G7 and G4 versus G8) for nimodipine and spironolactone (Fig. 1 and 2), the dissolution profiles were observed to be very similar, but they had faster and higher drug release rates than tablets obtained from physical mixtures (PM1 to PM 8). Within 15 minutes, at least 60\% of all of them had dissolved, notwithstanding the use of croscarmellose sodium or sodium dodecyl sulfate. 
A

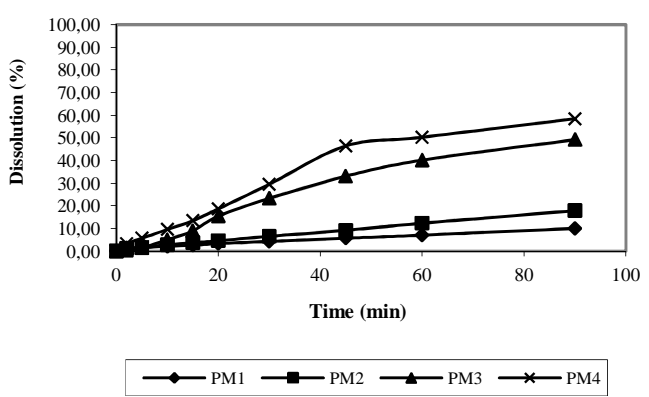

$\mathrm{C}$

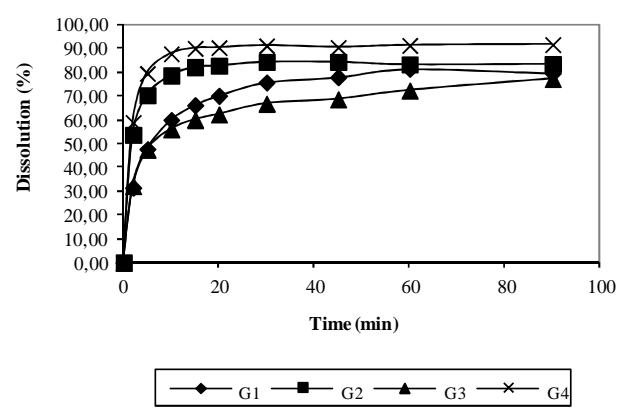

Spironolactone

B

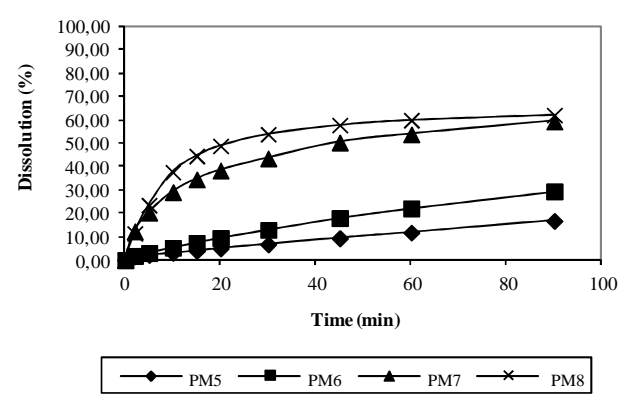

$\mathrm{D}$

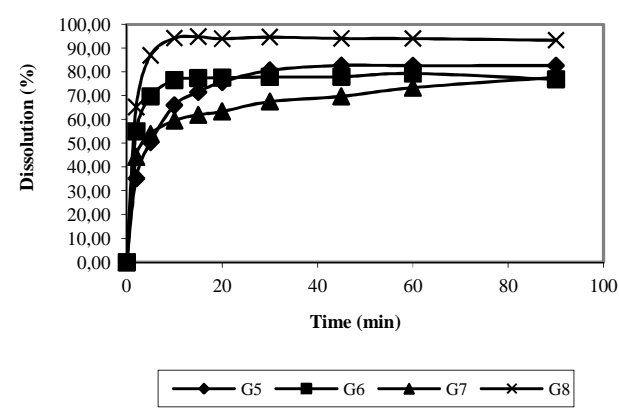

Figure 2 - Spironolactone dissolution profiles in $0.1 \mathrm{M}$ hydrochloride acid with $0.1 \%$ sodium dodecyl sulfate, using apparatus 1 at $100 \mathrm{rpm}$ for 90 minutes (PM= tablets obtained from physical mixtures, $\mathrm{G}=$ tablets obtained from granules).

It is not easy to obtain fast and high rates of drug release from tablets when they contain drugs with poor water-solubility, like nimodipine and spironolactone, as can be observed in the dissolution profiles of the tablets obtained from the physical mixtures (Figures 1 and 2). However, granulation in the fluid bed equipment, by spraying the dispersed drug, was capable of generating granules that, in a solid dosage form, presented a dissolution profile with more than $80.0 \%$ drug release within 20 minutes for nimodipine, and this was not related to the use of the disintegrant or the surfactant.

The dissolution efficiency (DE) was calculated for all formulations, and the results are presented in Table 2.

The analysis of variance of the DE values shows that the dissolution profiles are not similar for the formulations. The results of ANOVA are presented in Table 3 (nimodipine) and Table 4 (spironolactone), and the interaction plots in Figures 3 and 4.

The ANOVA results show that the amount of sodium dodecyl sulfate added for nimodipine, and the addition of croscarmellose sodium for spironolactone, did not significantly influence the DE values. However, a significant difference in dissolution efficiency for both drugs was related to the type of material processed (physical mixture or fluid bed granules), according to Tables 3 and 4 .

Considering that the use of disintegrants, such as croscarmellose sodium, in pharmaceutical formulations is a good strategy to facilitate the breaking down of tablets into smaller parts, thus allowing for faster drug release, it was thought that their addition would enhance the dissolution behavior of both drugs. However, only the physical mixtures were observed to be significantly affected by croscarmellose sodium. 
Table 2 - Dissolution efficiencies (DE) of nimodipine and spironolactone formulations (PM = tablets obtained from physical mixtures; $\mathrm{G}=$ tablets obtained from fluid bed granules).

\begin{tabular}{ccccc}
\hline & \multicolumn{3}{c}{ Dissolution efficiency (\%) } \\
\hline \multirow{2}{*}{ Formulation } & \multicolumn{2}{c}{ Nimodipine } & Spironolactone \\
\cline { 2 - 5 } & PM & G & PM & G \\
\hline 1 & 5.6 & 73.0 & 11.6 & 95.3 \\
2 & 9.3 & 81.0 & 17.0 & 94.8 \\
3 & 29.2 & 66.3 & 20.8 & 84.3 \\
4 & 37.3 & 88.5 & 53.1 & 84.5 \\
5 & 9.1 & 76.6 & 59.4 & 95.4 \\
6 & 16.9 & 76.1 & 64.8 & 94.9 \\
7 & 45.7 & 68.0 & 72.8 & 87.2 \\
8 & 52.4 & 91.9 & 79.3 & 84.2 \\
\hline
\end{tabular}

Table 3 - ANOVA (analysis of variance) for DE results of nimodipine.

\begin{tabular}{lccccc}
\hline \multicolumn{1}{c}{ Source } & DF & SS & MS & F & p-value \\
\hline Material & 1 & 7302.1 & 7302.1 & 23.77 & 0.001 \\
Surfactant & 3 & 242.5 & 80.8 & 0.26 & 0.850 \\
Disintegrant & 1 & 1952.5 & 1952.5 & 6.36 & 0.030 \\
Error & 10 & 3071.9 & 307.2 & & \\
Total & 15 & 12569.0 & & & \\
\hline
\end{tabular}

$\mathrm{DF}=$ degrees of freedom, $\mathrm{SS}=$ sum of square, $\mathrm{MS}=$ mean square.

Table 4 - ANOVA (analysis of variance) for DE results of spironolactone.

\begin{tabular}{lccccc}
\hline \multicolumn{1}{c}{ Source } & DF & SS & MS & F & p-value \\
\hline Material & 1 & 10809.8 & 10809.8 & 95.07 & 0.000 \\
Surfactant & 3 & 1590.0 & 1590.0 & 4.66 & 0.028 \\
Disintegrant & 1 & 134.9 & 134.9 & 1.19 & 0.302 \\
Error & 10 & 1137.0 & 1137.0 & & \\
Total & 15 & 13671.7 & & & \\
\hline
\end{tabular}

$\mathrm{DF}=$ degrees of freedom, $\mathrm{SS}=$ sum of square, $\mathrm{MS}=$ mean square.

The interaction plot graphs enable the analysis of two variables at a time for nimodipine (Fig. 3) and spironolactone (Fig. 4). Considering the addition of croscarmellose sodium and the type of material (Figures 3 and 4), it was observed that the DE values for tablets obtained from physical mixtures of nimodipine and spironolactone without croscarmellose sodium $(5.6,9.3,29.2$ and $37.3 \%$, for nimodipine and 11.6, 17.0, 20.8 and 53.1\%, for spironolactone) were improved after the addition of croscarmellose sodium $(9.1,16.9,45.7$ and $52.4 \%$ for nimodipine and 59.4, 64.8, 72.8 and $79.3 \%$ for spironolactone).

However the formulations obtained by granulation in the fluid bed equipment, after spraying the dispersed drug onto the powders, consistently showed better DE values, notwithstanding the use of croscarmellose sodium: the DE percentages in nimodipine tablets obtained from granules were $73.0,81.0,66.3$ and $88.5 \%$, and after the addition of croscarmellose sodium, they rose to 76.6, 76.1,
68.0 and $91.9 \%$ (Fig. 3). Similarly for spironolactone, the DE values obtained were 95.3, $94.8,84.3,84.5 \%$, and $95.4,94.9,87.2,84.2 \%$ with croscarmellose sodium (Fig. 4).

When the correlation between the amount of sodium dodecyl sulfate and the type of material is considered (Figures 3 and 4), the DE values of tablets obtained from physical mixtures for both nimodipine and spironolactone were observed to present increasing values according to the amount of surfactant added $(0,0.5,1.0$ or $2.0 \%)$. The use of surfactants is a formulation approach to enhance the dissolution properties of drugs with poor water-solubility, but their use is limited due to toxicity and side effects (Stegemann et al. 2007). For the tablets obtained from granules obtained by spraying the dispersed drug in the fluid bed equipment, the influence of sodium dodecyl sulfate disappears and all DE values are higher than the DE values for the tablets obtained from physical mixtures. 


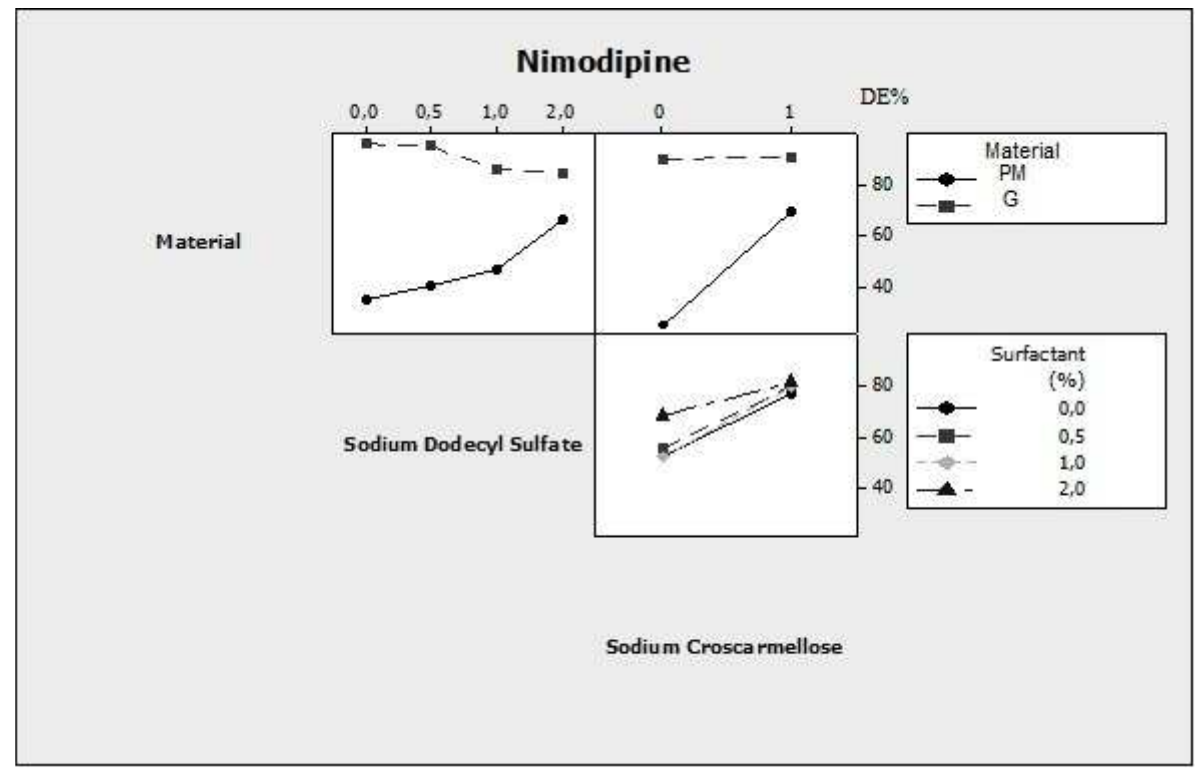

Figure 3 - Interaction plot (data means) for DE: nimodipine tablets obtained from physical mixtures (PM) and from granules $(\mathrm{G})$. DE\% = dissolution efficiency (y axis); concentrations of 0 to $2.0 \%, 0$ and $1 \%$, are from sodium dodecyl sulfate and sodium croscarmellose, respectively (x axis).

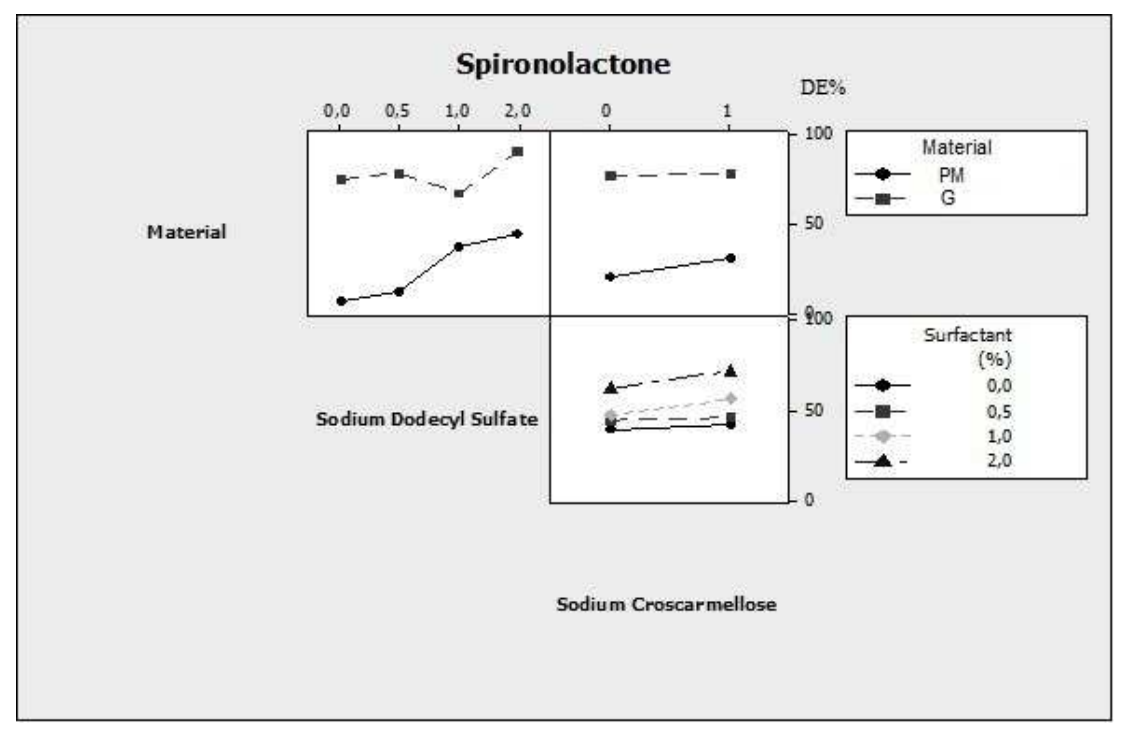

Figure 4 - Interaction plot (data means) for DE: spironolactone tablets obtained from physical mixtures (PM) and from granules (G). DE\% = dissolution efficiency (y axis); concentrations of 0 to $2.0 \%, 0$ and $1 \%$, are from sodium dodecyl sulfate and sodium croscarmellose, respectively (x axis).

The interaction plot of the surfactant and the disintegrant (Figures 3 and 4) shows that DE values for the tablets containing nimodipine, obtained from both physical mixtures and granules, are affected by the use of croscarmellose sodium. Regarding spironolactone, the DE values are affected by the amount of sodium dodecyl sulfate used. However, the resulting improvement of those excipients is no greater than the improvement obtained using the fluid bed granulation process. It is probable that fluid bed granulation, with the drug dispersed onto powder, 
leads to smaller and more uniform granules with better solubility properties in poorly water-soluble drugs, like nimodipine and spironolactone.

The addition of dispersed drugs is an interesting resource in fluid bed technology, because this procedure avoids the loss of drugs that may otherwise adhere to the walls of the processing equipment, especially in the upper sections, near the filters. By blowing the active agents directly onto the surface of the particles inside the bed, and in the presence of flocculant, granules are formed and these do not easily adhere to the walls of the equipment.

It is important to establish alternatives to enhance the dissolution rate of drugs with limited water solubility, notwithstanding the use of excipients, which can be toxic to humans. Fluid bed granulation has proved to be a process where the dissolution properties of water-insoluble drugs can be improved, without resorting to surfactants or disintegrants.

This study proves that fluid bed granulation can improve the dissolution rate of nimodipine and spironolactone tablets, without the use of other formulation strategies to enhance dissolution properties. The use of sodium dodecyl sulfate was more important to spironolactone, and croscarmellose sodium was able to improve the dissolution profile of nimodipine tablets. However, the impact of the use of the fluid bed was the most representative factor in both cases.

\section{REFERENCES}

Ansari MA, Stepanek F. The evolution of microstructure in three-component granulation and its effect on dissolution. Part Sci Technol. 2008; 26: 55 -66 .

Bouffard J, Kaster M, Dumont H. Influence of process variable and physicochemical properties on the granulation mechanism of mannitol in a fluid bed top spray granulator. Drug Dev Ind Pharm. 2005; 31, 923 $-933$.

British Pharmacopoeia, Her Majesty's Stationery Office, London, 2010, 2941 - 2942.
Fahmy RH, Kassem MA. Enhancement of famotidine dissolution rate through liquisolid tablets formulation: in vitro and in vivo evaluation. Eur J Pharm Biopharm. 2008; 69, 993 - 1003.

Iveson SM, Litster JD, Hapgood K, Ennis BJ. Nucleation, growth and breakage phenomena in agitated wet granulation process: a review. Powder Technol. 2001; 117, 3 - 39.

Leuner C, Dressman JB. Improving drug solubility for oral delivery using solid dispersions. Eur J Pharm Biopharm. 2000; 50, 47 - 60.

Lindenberg M, Kopp S, Dressman JB. Classification of orally administered drugs on the World Health Organization model list of Essential Medicines according to the biopharmaceutics classification system. Eur J Pharm Biopharm. 2004; 58, 265 - 278.

Murali Mohan Babu GV, Prasad ChDS, Ramana Murthy KV. Evaluation of modified gum karaya as carrier for the dissolution enhancement of poorly water soluble drug nimodipine. Int J Pharm. 2002; 234, $1-17$.

Papageorgiou GZ, Bikiaris D, Karavas E, Politis S, Docoslis A, Park Y, et al. Effect of physical state and particle size distribution on dissolution enhancement of nimodipine/PEG solid dispersions prepared by metl mixing and solvente evaporation. The AAPS $J$. 2006; 8, E623 - E631.

Stegemann S, Leveiller F, Franchi D, de Jong H, Lindén $\mathrm{H}$. When poor solubility becames an issue: from early stage to proof of concept. Eur J Pharm Sci. 2007; 31, 249 - 261.

Tardos GI, Khan MI, Mort PR. Critical parameters and limiting conditions in binder granulation of fine powders. Powder Technol. 1997; 94, 245 - 258.

United States Pharmacopeia (2009), 32 ed., United States Pharmacopeial Convention, Rockville MD, 3293 - 3594.

Vervaet C, Remon JP. Continuous granulation in pharmaceutical industry. Chem Eng Sci. 2005; 60, 3949 - 3957.

Vogt M, Kunath K, Dressman JB. Dissolution enhancement of fenofibrate by micronization, cogrinding and spray-drying: comparison with commercial preparations. Eur J Pharm Biopharm. 2008a; 68, $283-288$.

Vogt M, Kunath K, Dressman JB. Dissolution improvement of four poorly water soluble drugs by cogrinding with commonly used excipients. Eur $J$ Pharm Biopharm. 2008b; 68, 330 - 337. 